\title{
Communication \\ Excitation of Multi-Beam Interference and Whispering-Gallery Mode in Silica Taper-Assisted Polymer Microspheres for Refractometric Sensing
}

\author{
Huibo Fan ${ }^{1,2, *}$, Dawei Zhou ${ }^{1}$, Li Fan ${ }^{1}$, Yuanyan $\mathrm{Wu}^{1}{ }^{1}$, Hao Tao ${ }^{3}$ and Junbin Gong ${ }^{3}$ \\ 1 College of Physics Science and Technology, Yangzhou University, Yangzhou 225002, China; \\ MZ120180447@yzu.edu.cn (D.Z.); fanli@yzu.edu.cn (L.F.); yywu@yzu.edu.cn (Y.W.) \\ 2 Zhejiang Lab, Hangzhou 310000, China \\ 3 China Ship Development and Design Center, Wuhan 430064, China; aiar.th@stu.xjtu.edu.cn (H.T.); \\ hfan2@uottawa.ca (J.G.) \\ * Correspondence: hbfan@yzu.edu.cn
}

check for updates

Citation: Fan, H.; Zhou, D.; Fan, L.; $\mathrm{Wu}$, Y.; Tao, H.; Gong, J. Excitation of Multi-Beam Interference and Whispering-Gallery Mode in Silica Taper-Assisted Polymer Microspheres for Refractometric Sensing. Photonics 2021, 8, 117

https://doi.org/10.3390/

photonics 8040117

Received: 27 February 2021

Accepted: 7 April 2021

Published: 10 April 2021

Publisher's Note: MDPI stays neutra with regard to jurisdictional claims in published maps and institutional affiliations.

Copyright: (c) 2021 by the authors. Licensee MDPI, Basel, Switzerland. This article is an open access article distributed under the terms and conditions of the Creative Commons Attribution (CC BY) license (https:// creativecommons.org/licenses/by/ $4.0 /)$.

\begin{abstract}
We propose and numerically analyze a fiber-optic sensor based on a silica taper-assisted multiple polymer microspheres to realize high-sensitivity refractometric sensing due to the excitation of multi-beam interference and whispering-gallery modes (WGMs) in each microsphere. Up to 5 UVcured adhesive microspheres are dripped in sequence on the $2 \mu \mathrm{m}$-diameter silica taper with the certain distances in between. Scattering and reflection of light in each microsphere enhance the multibeam interference because of the emergence of high-order modes. Moreover, WGMs with certain resonant wavelengths are excited in the microspheres, further enhancing the transmission spectrum with high contrast and quality factor. Furthermore, irregular transmission spectrum corresponds to the large wavelength tuning range. Propagating light between the microspheres could be strongly affected by the environmental factor with the large transmission spectrum shift, therefore realizing the refractometric sensing with high sensitivity of $846 \mathrm{~nm} / \mathrm{RIU}$ based on the taper-assisted 2-spheres sensor. Furthermore, another two microspheres are placed on both sides of 2-spheres sensor to form 4-spheres-loop sensor, re-collecting those large-angle scattering light with the enhancement of multi-beam interference and WGMs. Therefore, the sensitivity of 4-shperes-loop sensor is further improved to the maximum of $1296.3 \mathrm{~nm} / \mathrm{RIU}$.
\end{abstract}

Keywords: fiber-optic sensor; multi-beam interference; whispering-gallery mode; refractometric sensing

\section{Introduction}

Refractometric sensors have attracted of great interest in diverse applications such as environmental monitoring, biosensing, and safety monitoring [1-4]. Fiber-optic sensors show unique advantages of compact structure, immunity to electromagnetic interference, and long-distance measurement, which have been used to sense many environmental factors, including static measurements of temperature [5-8], strain [9-11], and refractive index (RI) [12-14], and dynamic measurement of ultrasound [15-18]. Fiber-optic RI sensors have been deeply studied based on various structures, such as Fabry-Perot interferometer (FPI) [12-14], Mach-Zehnder interferometer (MZI) [19,20], fiber Bragg grating (FBG) [21,22], and surface plasmon polariton (SPP) [23-25]. For example, tilted fiber Bragg grating is used for the concentration sensing [26]; stretchable multiplexed optical fiber is used for the multimodal wearable sensing [27]; multi-core fiber sensor is used for the cancer cells detection [28]. Especially, FPI based RI sensors are usually the open-cavity or sealed-cavity structures with the relatively complicated fabrication process of laser micromachining or chemical etching [12,14]. The sensitivity of an open-cavity structure is larger than that of a sealed-cavity structure because of the high amount of light propagating outside the optical fiber that shows a strong interaction with the environmental factors. The MZI based RI 
sensor, often containing many fiber components such as single-mode fibers, couplers, and attenuator, has large size to realize the refractometric sensing [19]. FBG with fabrication process of laser micromachining requires relatively complicated manipulation of laser focusing and power, while SPP structure with metal film deposition also requires complex control on the film thickness and position.

Another potential of small-diameter silica microfiber with strong evanescent field has high sensitivity to respond the environmental change, showing great interest in the refractometric sensing [29-31]. Few modes, including fundamental mode (LP01) and second-order mode (LP02), propagate in the microfiber to realize the relatively weak multimode interference, demonstrating the transmission spectrum with relatively low contrast. In addition, most optical light transmits inside the microfiber while only a fraction of light outside the microfiber due to the evanescent field effect. Therefore, enhancing the multimode interference in the microfiber and increasing the optical power outside the microfiber are a potential direction to realize the high-sensitivity refractometric sensing. Integrating a high-RI microsphere on the silica taper can enhance the scattering and reflection in the microsphere, subsequently enhancing the multi-mode interference due to the emergence of high-order modes. Furthermore, cascaded microspheres on silica taper are the good potential to realize high-sensitivity refractometric sensing because of the enhanced multimode interference in each microsphere and the high level of propagating light between microspheres interacting strongly with the environmental factors.

In this paper, we designed and numerically analyzed the cascaded adhesive microspheres dripped on a $2 \mu \mathrm{m}$-diameter silica taper to realize the high-sensitivity refractometric sensing. Up to $5 \mathrm{UV}$-cured adhesive microspheres are dripped in sequence on the silica taper with certain distances in between. Scattering and reflection of light in each microsphere enhance the multi-beam interference because of the emergence of high-order modes. Moreover, WGMs with certain resonant wavelengths are excited in the microspheres, inducing the high-contrast irregular transmission spectrum with large wavelength tuning range. The majority of propagating light between the microspheres could be strongly influenced by the environmental RI change, along with the large spectrum shift, subsequently showing the refractometric sensing with the sensitivity up to $846 \mathrm{~nm} / \mathrm{RIU}$ based on taper-assisted 2-spheres sensor. Furthermore, another two microspheres are placed on both sides of 2-spheres sensor to form 4-spheres-loop sensor, re-collecting those large-angle scattering light with the excitation of multi-beam interference and WGMs, which demonstrate the sensitivity improvement to the maximum of $1296.3 \mathrm{~nm} / \mathrm{RIU}$. Therefore, this proposed design provides a simple and flexible method to tune many parameters for the optimization of the device performance, such as the factors of tapered fiber's diameter, microspheres' diameters, and the corresponding distances in between. Tapered fiber-assisted multiple microspheres are the good platform for the high-sensitivity sensing, including refractometric, strain, and temperature sensing.

\section{Materials and Methods}

Figure 1a shows the schematic diagram of $2 \mu \mathrm{m}$-diameter silica taper-assisted $5 \mathrm{UV}$ cured adhesive microspheres. Before the simulation process, it is feasible in the expected fabrication procedure, silica microfiber could be fabricated with the certain waist diameter and waist length by using the fiber-drawing system [18]. The tapered fiber with such small diameter is feasible, which has been realized in our previous references [32,33] to detect the ultrasound signal or couple the microcavity. Furthermore, small fiber diameter could reduce the surface tension of the tapered fiber and is useful to support the adhesive microsphere. A UV adhesive droplet with refractive index of 1.54 and a certain volume is supported by another silica taper and transferred forward the waist region of microfiber. After that, UV light is used to cure the adhesive microsphere. Multiple microspheres could be transferred and cured on the silica microfiber in sequence to realize cascadedmicrospheres sensor. In addition, the adhesive viscosity could be changed by using the UV light irradiation for a suitable time before transferring the drop onto the tapered fiber. 
Small fiber diameter and proper adhesive viscosity are useful for the realization of adhesive microspheres with the perfect shape on the tapered fiber. Therefore, this compact fiber-optic sensor is feasible in the proposed future experiment, which this transferring technique has been shown in our previous paper about the silica taper-assisted dual micro-bubbledevice for the ultrasound detection [34]. The inset in Figure 1a is the cross section of first two microspheres with diameters of $D_{1}$ and $D_{2}$, and gap ${ }_{1}$ in between. Input light first propagates through the first microsphere, resulting in the light scattering and reflection in the adhesive microsphere due to its higher refractive index than that of silica microfiber. The majority of scattering light reflects back to the microsphere, along with the refraction outside the microsphere. The scattering light with the emergence of high-order modes in the first microsphere enhances the multi-beam interference, associated with the excitation of WGMs with certain resonant wavelengths. The detailed description of light propagating in the adhesive microbubble with the reflection and refraction has been shown in the reference [34]. The combination of multi-beam interference and WGMs promotes the highcontrast transmission spectrum, while multi-beam interference is in the primary status because only few part of scattering light with the special wavelengths has the possibility to achieve the WGMs in the adhesive microsphere. Furthermore, the refraction light from the first microsphere continues to spread forward the second microsphere to further excite the high-order modes and WGMs, associated with the same physical mechanism and light intensity attenuation in the subsequent microspheres.

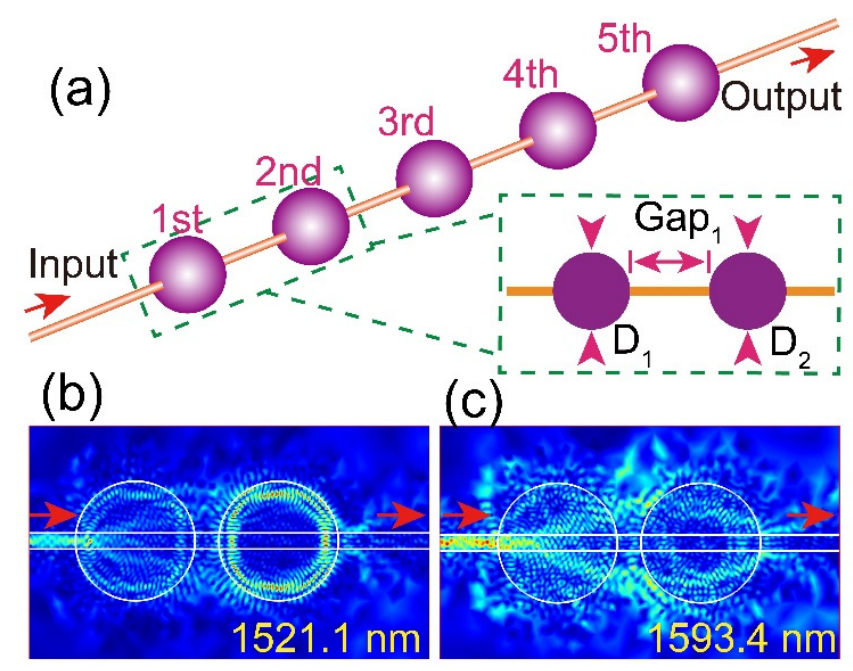

Figure 1. (a) The proposed scheme of silica taper-assisted 5 UV-cured adhesive microspheres. Inset shows the cross section of first two microspheres with diameters of $D_{1}$ and $D_{2}$, and gap in $_{1}$ between. $(\mathbf{b}, \mathbf{c})$ Electric field distributions of fiber-optic 2-spheres sensor with $D_{1}=D_{2}=15 \mu \mathrm{m}$, gap $1=3 \mu \mathrm{m}$, and different input wavelengths.

In the simulation process, two-dimensional finite element method based on COMSOL Multiphysics software is used to numerically analyze the light scattering, reflection, and refraction in the taper-assisted cascaded microspheres. The surrounding layer in the simulation is the perfectly matched layer. The diameter of silica taper is fixed to $2 \mu \mathrm{m}$ with the analysis of influence of adhesive microspheres on the device transmission spectrum for simplicity. The input light is set to a broadband source with the input power of $100 \mathrm{~mW}$, ranging from 1500 to $1600 \mathrm{~nm}$, without considering its polarization due to the random scattering light in all directions. As input light has the special wavelength, light can propagate along the inner wall of adhesive microsphere due to the total reflection effect, corresponding to the whispering-gallery mode. For example, for the case of $1521.1 \mathrm{~nm}$, propagating light with the clear total reflection can be observed in the right-side microsphere in Figure 1b, which is the whispering-gallery mode. Two microspheres have the same diameter of $15 \mu \mathrm{m}$ and distance of $3 \mu \mathrm{m}$ in between. Scattering and reflection of light occupy the primary status as 
input wavelength at $1593.4 \mathrm{~nm}$, as shown in Figure 1c. The coupling efficiency of refraction light from the first microsphere into the second microsphere strongly depends on the refractive index of surrounding environment, because of the angle variation of refraction light, finally benefitting to the high-sensitivity RI sensing. Before analyzing the RI sensing, the transmission spectrum of the hybrid structure is first analyzed and optimized by changing the microspheres' diameters, gap distances in between and the number of microspheres in sequence.

\section{Results and Discussion}

The transmission spectrum of silica taper-assisted only one microsphere is analyzed first under different diameters $(D)$, as shown in Figure 2a, along with the corresponding comparisons of the contrast (highlighted dips in Figure $2 a$ ) and quality factor (Q) in Figure $2 b$. The contrast of up to $12.7 \mathrm{~dB}$ is obtained as $D=15 \mu \mathrm{m}$. When $D$ is small, for example less than $15 \mu \mathrm{m}$, input light scatters and then reflects at the microsphere interface with small reflection angle, accompanying by a small number of reflection in the microsphere. Therefore, multi-beam interference is relatively weak with the emergence of few high-order modes, corresponding to the low contrast of transmission spectrum. As $D$ is large, such as $20 \mu \mathrm{m}$, the corresponding large reflection angle results in a large number of reflection in the microsphere with the emergence of huge high-order modes. However, increased propagating loss in large microsphere induces the intensity decrease of each high-order modes, showing the weak multi-mode interference. The quality factors of all patterns in the transmission of the whole cases are calculated and then plotted the maximal values in Figure $2 b$, simplified as $Q$ (signing the same in the following investigation). It is shown that the maximal value of quality factor is 704 as $D=15 \mu \mathrm{m}$, placed at the same case with that of contrast. Figure 2c,d clearly demonstrates the strong scattering in $15 \mu \mathrm{m}$-diameter microsphere while weak effect in $20 \mu \mathrm{m}$-diameter microsphere. In addition, the reduced transmission in Figure 2a also verifies the increase of propagating loss as the microsphere diameter is increased.
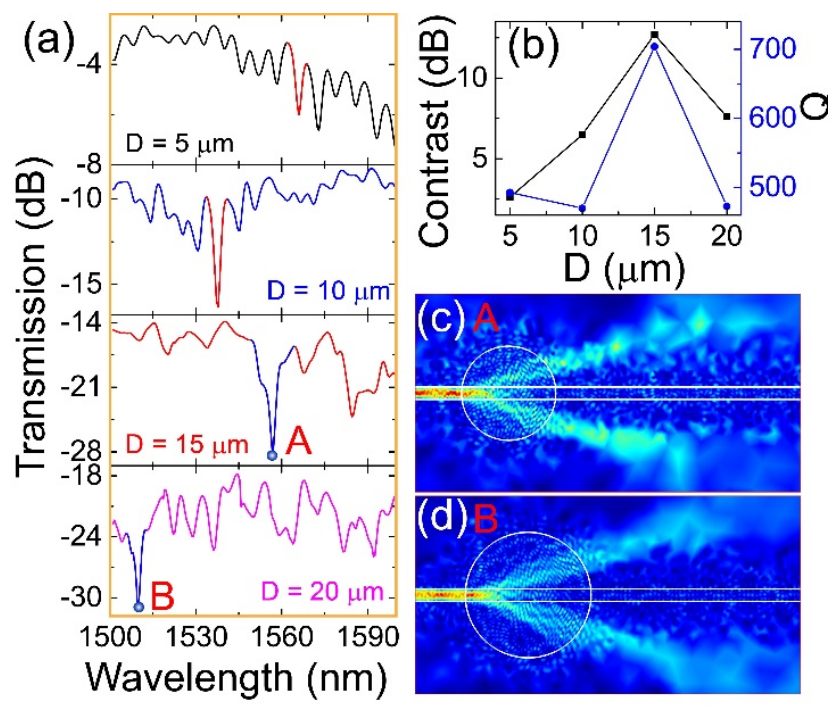

Figure 2. (a) The transmission spectra of silica taper-assisted only one microsphere under different $D$. (b) The corresponding comparisons of the contrast and quality factor (Q) under different $D$. (c,d) Electric field distributions at point A and point B in Figure 2a.

After that, the transmission spectrum of silica taper-assisted two microspheres with the equal diameter of $15 \mu \mathrm{m}$ is analyzed under different gap in between, as shown in Figure 3a, along with the corresponding comparisons of the contrast (highlighted dips in Figure $3 a$ ) and the quality factor in Figure $3 b$. The maximal contrast and $Q$ are $27.3 \mathrm{~dB}$ and 850 , respectively, when gap $=6 \mu \mathrm{m}$, which the electric field distributions at different input 
wavelengths of point $A$ and point $B$ are demonstrated in Figure $3 c, d$, respectively. For point A of $1514.6 \mathrm{~nm}$, input light disperses in the first microsphere and then converges in the right side of second microsphere to realize the multi-beam interference. For point $B$ of $1557.8 \mathrm{~nm}$, in addition to some part of scattering light with the multi-beam interference, WGMs are excited due to the certain resonant wavelength in both microspheres, therefore obtaining the maximal contrast of $27.3 \mathrm{~dB}$. Furthermore, refraction light from the first microsphere has the relatively critical propagating angle to couple into the second microsphere as gap $=6 \mu \mathrm{m}$, realizing the combination of multi-beam interference and WGMs. As gap is small, for example gap $=3 \mu \mathrm{m}$, only WGMs are generated as shown in Figure $1 \mathrm{~b}$ with contrast of $3.7 \mathrm{~dB}$, or only multi-beam interference is produced as shown in Figure 1c with contrast of $23 \mathrm{~dB}$ due to improper propagating angle of refraction light coupling into the second microsphere. As gap is large, such as gap $=9 \mu \mathrm{m}$, the propagating loss increase of refraction light results in the weak intensity in the second microsphere, showing the weak multi-beam interference and WGMs with small contrast and low quality factor of transmission spectrum. In addition, considering tapered fiber passing through the adhesive microsphere, input light that propagates in the tapered fiber could scatter in all directions in the adhesive microsphere. Some special scattering light can meet the condition of total internal reflection, finally exciting the WGMs in the adhesive microsphere, which it can be regarded as the strongly coupled light in the microsphere.

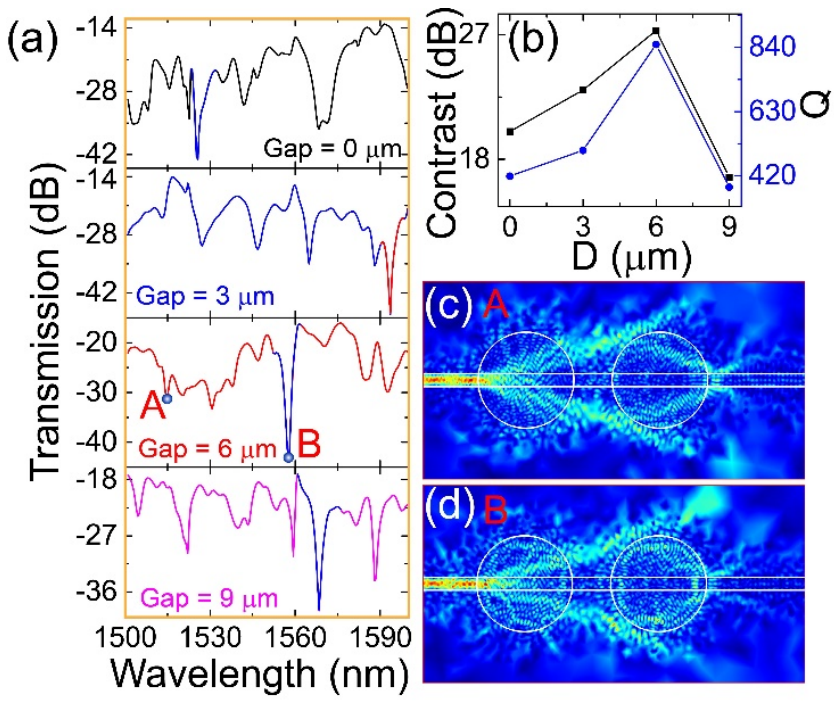

Figure 3. (a) The transmission spectra of silica taper-assisted two microspheres with the equal diameter of $15 \mu \mathrm{m}$ under different gap in between. (b) The corresponding comparisons of the contrast and quality factor under different gap. (c,d) Electric field distributions at point A and point B in Figure 3a.

Third, the transmission spectrum of silica taper-assisted two microspheres with $D_{1}=15 \mu \mathrm{m}$ and gap $=6 \mu \mathrm{m}$ is analyzed under different $D_{2}$, as shown in Figure $4 \mathrm{a}$, along with the corresponding comparisons of the contrast and quality factor in Figure $4 \mathrm{~b}$. As two microspheres have the same diameter of $15 \mu \mathrm{m}$, combination of multi-beam interference and WGMs induces the maximal contrast and quality factor of $27.3 \mathrm{~dB}$ and 850 , respectively. As the diameter of second microsphere is small, only few refraction light couples into the second microsphere, as shown the electric field distribution in Figure 4c, demonstrating the weak multi-beam interference and WGMs with the small-contrast and low-Q transmission spectrum. As the diameter of second microsphere is large, such as $D_{2}=25 \mu \mathrm{m}$, refraction light from the first microsphere would refracts into the second microsphere, associated with the weak multi-beam interference, as shown the electric field distribution in Figure 4d. Based on the analyses in Figures 2-4, it could be concluded that silica taper-assisted two microspheres with $D_{1}=D_{2}=15 \mu \mathrm{m}$ and gap $=6 \mu \mathrm{m}$ have the maximal contrast and quality factor 
of transmission spectrum due to the combination of strong multi-beam interference and WGMs. In the following investigation, the diameters of multiple microspheres are fixed to $15 \mu \mathrm{m}$ with the same gap of $6 \mu \mathrm{m}$ in between.

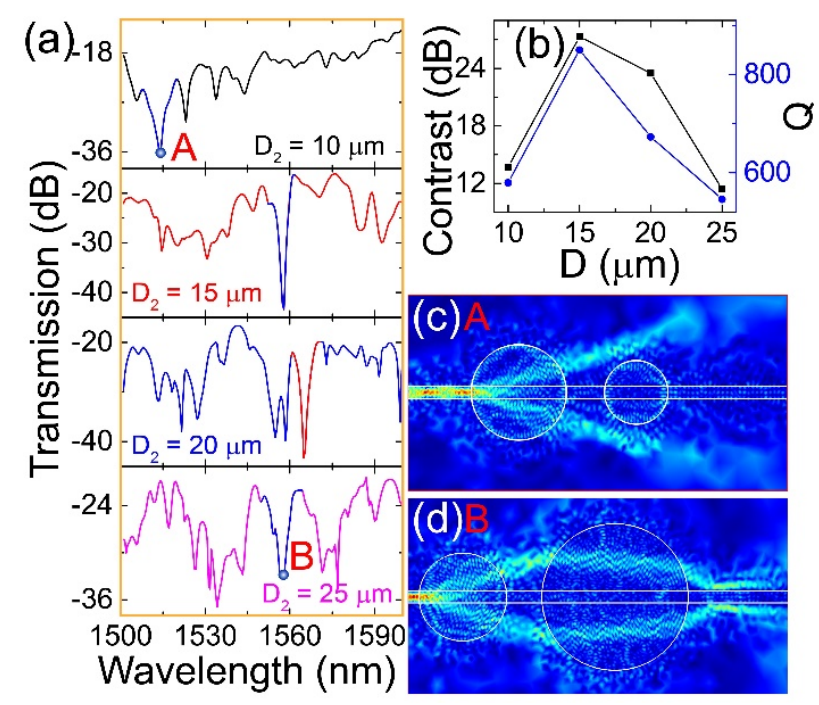

Figure 4. (a) The transmission spectra of silica taper-assisted two microspheres with $D_{1}=15 \mu \mathrm{m}$ and gap $=6 \mu \mathrm{m}$ under different $D_{2}$. (b) The corresponding comparisons of the contrast and quality factor under different $D_{2}$. (c,d) Electric field distributions at point A and point B in Figure 4a.

The influence of the number of microspheres on the transmission spectrum of silica taper-assisted multiple microspheres with the equal diameter of $15 \mu \mathrm{m}$ and equal gap of $6 \mu \mathrm{m}$ is also analyzed, as shown in Figure 5a, including the cases of three microspheres (3S), four microspheres (4S), and five microspheres (5S), respectively. The corresponding comparisons of contrast and quality factor are demonstrated in Figure 5e. More microspheres on the silica taper have not the contribution on the enhancement of multi-beam interference and WGMs; the optimal condition with the highest contrast and quality factor shows at the case of two microspheres (2S) with the same diameter of $15 \mu \mathrm{m}$ and gap of $6 \mu \mathrm{m}$ in between, as described in Figure 5e. Figure $5 \mathrm{~b}-\mathrm{d}$ demonstrate the electric field distributions at points A, $\mathrm{B}$, and $\mathrm{C}$, corresponding to the cases of $3 \mathrm{~S}, 4 \mathrm{~S}$, and $5 \mathrm{~S}$, respectively. It is clearly shown that multi-beam interference and WGMs primarily distribute in the first three microspheres. The later microspheres can be regarded as the perturbation to increase the propagating loss of high-order modes, although accompanied by the emergence of some new high-order modes with weak intensity. As the number of microsphere increases, the intensity of multi-beam interference and WGMs would be weakened due to the increased propagating loss, along with the decreases of contrast and quality factor in the transmission spectrum. In addition, as shown in Figure 5a, only few dips exist in the case of $4 \mathrm{~S}$ relative to more dips in that of 3S, showing that the disappearance of many high-order modes is in the primary status in the fourth microsphere due to the increased propagating loss. The latter microspheres in the proposed device could be regarded as the enhanced factors for the improvement of multi-beam interference and also as the filters to reduce the high-order modes in the transmission spectrum. However, the emergence of many new high-order modes is in the advantage position in the fifth microsphere, demonstrating more dips in the case of $5 S$. 

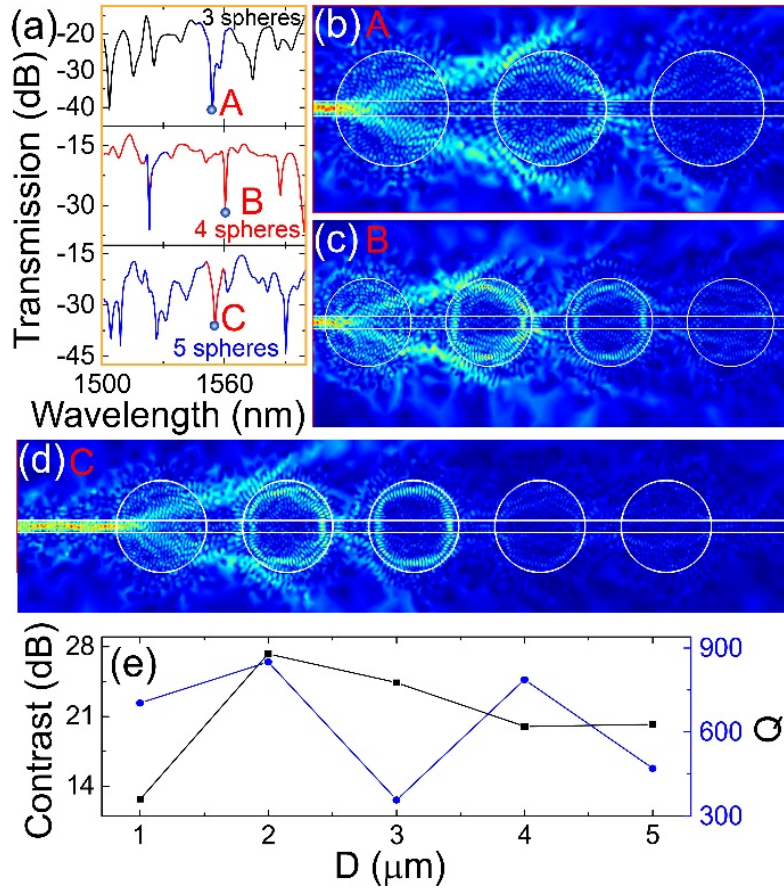

Figure 5. (a) The transmission spectra of silica taper-assisted multiple microspheres with $D=15 \mu \mathrm{m}$ and gap $=6 \mu \mathrm{m}$ under different number of microspheres. (b-d) Electric field distributions at points A, B and C in Figure 5a. (e) The corresponding comparisons of the contrast and quality factor under different number of microspheres.

Considering that most of the scattering light propagating outside the microspheres, accompanied by the transmission spectrum with high contrast and irregular dips, this fiber-optic sensor would be benefit for the high-sensitivity refractometric sensing. In the expected experiment, fiber-optic sensor could be placed in the vacuum chamber with RI of 1.0 as an example. As the gas pressure of the chamber is changed, refractive index of the surrounding environment around the fiber-optic sensor varies subsequently, resulting in the effective RI change of fiber-optic sensor along with the transmission spectrum shift. By calculating the corresponding wavelength shift with the refractive index change, the RI sensitivity $(\mathrm{S}=\delta \lambda / \delta \mathrm{n})$ can be achieved. The effective RIs of high-order modes depend on the energy contribution in the tapered fiber, microspheres, and surrounding medium. The increased surrounding RI results in the increased effective RI, demonstrating the transmission spectrum shifting to the longer wavelength, as shown the case of $2 \mathrm{~S}$ as an example in the Figure 6a. The refractometric properties of 5 proposed sensors with different number of microspheres are shown in Figure $6 \mathrm{~b}$. Due to the combination of multi-beam interference and WGMs, irregular dips in the transmission spectrum results in the large wavelength tuning range, associated with the high sensitivity. This is because each mode in the microspheres has special effective refractive index and certain optical sensitivity. The combined contribution of all high-order modes in the proposed device results in the high refractometric sensitivity. In Figure $6 c$, high sensitivity up to $846 \mathrm{~nm} / \mathrm{RIU}$ is obtained based on the silica taper-assisted two microspheres (2S), following the sensitivity reduction as the number of microsphere increases. As analyzed in Figure 5, when more cascaded microspheres are added in the silica taper, the majority of the power of the input light would be lost in the long-length propagation, corresponding to the intensity decrease of many high-order modes in this hybrid structure. Therefore, multi-beam interference and WGMs would be weakened, associated with the decreases of contrast and quality factor of transmission spectrum, corresponding to the sensitivity decrease in the refractive index sensing. 

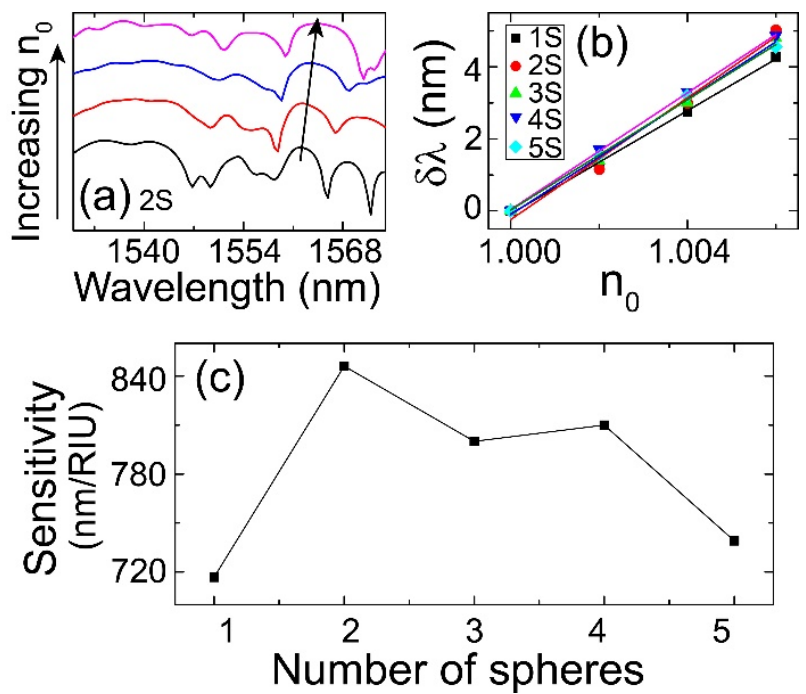

Figure 6. (a) The transmission spectrum shift of taper-assisted two microspheres (2S) as an example as increasing the surrounding refractive index $\left(\mathrm{n}_{0}\right)$. (b) The wavelength shifts of 5 proposed sensors versus the changes of surrounding RI. (c) The sensitivity comparison of 5 proposed sensors, simply signed as number of spheres.

In addition, this fiber-optic sensor is feasible and could be easily and accurately realized based on the expected transferring technique [34]. Although there are many references about the arrays of microspheres, few literatures have reported the tapered fiber-assisted adhesive microspheres for the refractometric sensing. For example, chains of $300 \mu \mathrm{m}$ sapphire spheres with index of 1.77 or $5 \mu \mathrm{m}$ polystyrene microspheres are used for the periodical focusing of light $[35,36]$. $\mathrm{BaTiO}_{3}$ microspheres or polystyrene microspheres are applied for super-resolution microscopy $[37,38]$. Furthermore, these microspheres are all obtained from the commercial companies, such as Cospheric Corp. and Duke Scientific Corp., and have the specified dimensions associated with few flexibility, which are difficult to integrate with the optical fiber. Based on melting and splicing single-mode fibers, several silica microspheres are fused together for the strain sensing [39], while large-diameter microspheres must be fused and fixed together, along with the nonadjustable distance in between.

As optical light illuminates the silica taper-assisted two microspheres, the majority of light propagates with the large scattered angle, without contributing to multi-beam interference and WGMs, as shown in Figure 3c,d, subsequently no impact on the high-sensitivity refractometric sensing. It can be predicted that the sensitivity would be increased when the large-angle propagating light couples into new microspheres to further enhance the multibeam interference and WGMs. As described the schematic setup in Figure 7a, an optimized structure is designed to enhance the multi-mode interference and then increase the corresponding sensitivity of refractive index sensing. The hybrid sensor of silica taper-assisted two microspheres is placed on the $\mathrm{MgF}_{2}$ substrate. Another two adhesive microspheres (S3 and S4) are transferred on both sides of 2-spheres sensor to form 4-spheres-loop sensor, then re-collecting the large-angle scattering light to excite the multi-beam interference and WGMs in S3 and S4. In the simulation process, $D_{1}=D_{2}=15 \mu \mathrm{m}$ and gap $=6 \mu \mathrm{m}$, along with the change of diameters of S3 and S4 $\left(D_{3}=D_{4}=D_{3,4}\right)$, as shown the inset in Figure 7a. Figure $7 \mathrm{c}-\mathrm{e}$, demonstrate the corresponding electric field distributions at points $\mathrm{A}, \mathrm{B}$ and $\mathrm{C}$ in Figure $7 \mathrm{~b}$ that is the transmission spectra under different $D_{3,4}$. For the case of four microspheres with the same diameter of $15 \mu \mathrm{m}$, as described the red curve of transmission spectrum in Figure 7b, it has the maximal contrast of $25.6 \mathrm{~dB}$ (as shown in Figure 8a), which is similar to the case of 2-spheres sensor with $D_{1}=D_{2}=15 \mu \mathrm{m}$ and gap $=6 \mu \mathrm{m}$ in Figure 5e. However, as demonstrated in Figure 8b, the maximal quality factor of 1481 is higher than that of 850 as shown in Figure 5e, showing the further enhanced multi-beam interference 
and WGMs in S3 and S4. As $D_{3,4}$ is small, such as $9 \mu \mathrm{m}$, the electric field distribution at point A shows small scattering light in S3 and S4 with weak multi-beam interference and WGMs, simultaneously producing a high propagating loss due to scattering light in different angles. As $D_{3,4}$ is large, such as $21 \mu \mathrm{m}$, strong scattering light and large propagating loss also result in the weak multi-beam interference and WGMs in S3 and S4, finally weakening the emergence of high-order modes in S2. Therefore, the contrasts and quality factors of transmission spectra in the cases of $D_{3,4}=9 \mu \mathrm{m}$ and $21 \mu \mathrm{m}$ are smaller than those of the case of $D_{3,4}=15 \mu \mathrm{m}$.

(a)
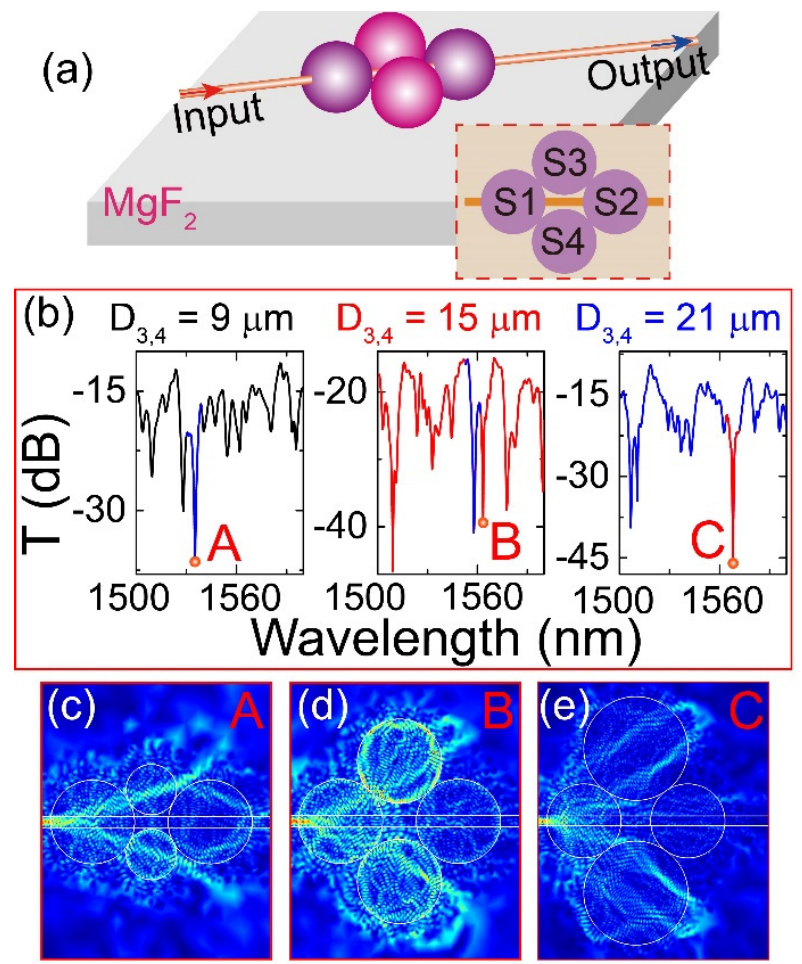

Figure 7. (a) Schematic of silica taper-assisted 4-spheres-loop sensor. Inset shows the cross section of 4-spheres-loop (S1, S2, S3, and S4). (b) The transmission (T) spectra of 4-spheres-loop sensor with $D_{1}$ $=D_{2}=15 \mu \mathrm{m}$ and gap $=6 \mu \mathrm{m}$ under different diameters of S3 and S4 $\left(D_{3}=D_{4}=D_{3,4}\right)$. (c-e) Electric field distributions at points A, B and C in Figure $7 \mathrm{~b}$.
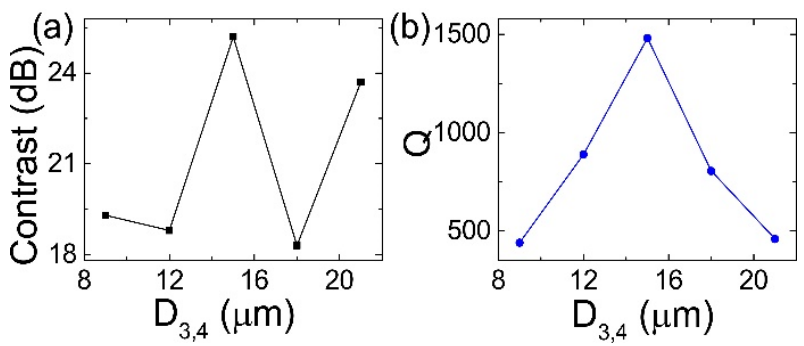

Figure 8. (a,b) The corresponding variations of the contrast and quality factor of 4-spheres-loop sensors undet different $D_{3,4}$.

As the surrounding RI of 4-spheres-loop sensor is increased, transmission spectrum shifts to the longer wavelength, as shown the case of $D_{3,4}=15 \mu \mathrm{m}$ in Figure 9a. The corresponding RI properties of 4-spheres-loop sensors under different $D_{3,4}$ are demonstrated in Figure $9 b, c$, showing the maximal sensitivity of $1296.3 \mathrm{~nm} / \mathrm{RIU}$ based on the 4-spheres-loop sensor with $D_{3,4}=15 \mu \mathrm{m}$. When $D_{3,4}$ is smaller or larger than $15 \mu \mathrm{m}$, those WGMs formed in S1 could not couple into S3 and S4, therefore weaking the intensity of high-order modes. 
Furthermore, large propagation loss of scattering light in different angles also results in the intensity weakening of multi-beam interference and WGMs, finally inducing the lower contrast and quality factor, as shown in Figures 7 and 8. Comparing to $846 \mathrm{~nm} / \mathrm{RIU}$ of 2-spheres sensor (2S) in Figure 6, the sensitivity of 4-spheres-loop sensor with equal diameter of $15 \mu \mathrm{m}$ has great improvement by adding another two microspheres on both sides of 2-spheres sensor to enhance the multi-beam interference and WGMs. For the performance comparison, several types of fiber-optic refractometric sensors are summarized, including the configurations of FPI, MZI and FBG, as shown in Table 1. Our designed fiber taper-assisted microspheres demonstrate well sensing performance of $1296.3 \mathrm{~nm} / \mathrm{RIU}$ relative to those existing refractometric sensors. This proposed method presents a flexible and simple way to tune many parameters for the optimization of the device performance, finally achieving the high-sensitivity refractometric sensing.

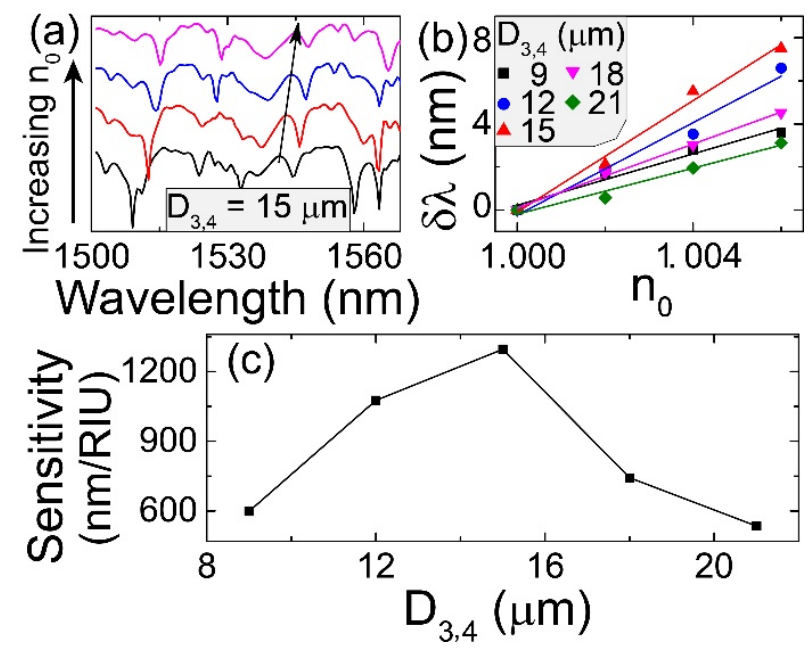

Figure 9. (a) The transmission spectrum shift of taper-assisted 4-spheres-loop sensor as increasing $\mathrm{n}_{0}$. (b) The wavelength shifts with different $D_{3,4}$ versus the changes of surrounding RI. (c) The sensitivity comparison of 4-spheres-loop sensor under different $D_{3,4}$.

Table 1. Comparison of the sensing performance of the fiber-optic refractometric sensors.

\begin{tabular}{ccc}
\hline Configuration & Sensitivity $(\mathbf{n m} / \mathbf{R I U})$ & Reference \\
\hline FPI & 994 & {$[12]$} \\
FPI & 1097 & {$[13]$} \\
FPI & 1163 & {$[14]$} \\
MZI & -93.2429 & {$[19]$} \\
MZI & 1426.7 & {$[20]$} \\
FBG & 100 & {$[21]$} \\
FBG & 312.5 & {$[22]$} \\
Taper-assisted microspheres & 1296.3 & This work \\
\hline
\end{tabular}

\section{Conclusions}

In summary, silica taper-assisted multiple polymer microspheres are numerically investigated to realize the high-sensitivity refractometric sensing. Scattering and reflection of light in each microsphere enhance the multi-beam interference, accompanied by the excitation of WGMs with certain resonant wavelengths, further enhancing the transmission spectrum with high contrast and large quality factor. Propagating light between the microspheres could be strongly affected by the environmental factor with the large transmission spectrum shift, therefore realizing the refractometric sensing with high sensitivity of $846 \mathrm{~nm} / \mathrm{RIU}$ based on 2-spheres sensor with equal diameter of $15 \mu \mathrm{m}$. Furthermore, another two microspheres, placed on both sides of 2-spheres sensor, realize the 4-spheres-loop sensor, re-collecting the large-angle scattering light with the enhancement of multi-beam 
interference and WGMs. Therefore, the sensitivity of 4-shperes-loop sensor is further improved to the maximum of $1296.3 \mathrm{~nm} / \mathrm{RIU}$. This novel proposal is a good reference for the compact ultra-sensitive fiber-optic sensor.

Author Contributions: Conceptualization, H.F.; methodology, H.F.; software, H.F. and D.Z.; validation, H.F. and D.Z.; formal analysis, H.F., L.F., Y.W., H.T. and J.G.; investigation, D.Z.; resources, H.F.; data curation, H.F. and D.Z.; writing—original draft preparation, H.F; writing—review and editing, H.F.; visualization, H.F.; supervision, H.F.; project administration, H.F.; funding acquisition, H.F., L.F., Y.W. and H.T. All authors have read and agreed to the published version of the manuscript.

Funding: This research was funded by National Natural Science Foundation of China, grant number 11804292, grant number 11774301, grant number 11705160 and grant number 11705160.

Data Availability Statement: Not applicable.

Conflicts of Interest: The authors declare no conflict of interest.

\section{References}

1. James, S.W.; Korposh, S.; Lee, S.; Tatam, R.P. A long period grating-based chemical sensor insensitive to the influence of interfering parameters. Opt. Express 2014, 22, 8012-8023. [CrossRef]

2. Wang, X.-D.; Wolfbeis, O.S. Fiber-Optic Chemical Sensors and Biosensors (2013-2015). Anal. Chem. 2016, 88, 203-227. [CrossRef] [PubMed]

3. Yin, M.-J.; Gu, B.; An, Q.-F.; Yang, C.; Guan, Y.L.; Yong, K.-T. Recent development of fiber-optic chemical sensors and biosensors: Mechanisms, materials, micro/nano-fabrications and applications. Coordin. Chem. Rev. 2018, 376, 348-392. [CrossRef]

4. Díaz, C.A.R.; Leal-Junior, A.; Marques, C.; Frizera, A.; Pontes, M.J.; Antunes, P.F.C.; André, P.S.B.; Ribeiro, M.R.N. Optical Fiber Sensing for Sub-Millimeter Liquid-Level Monitoring: A Review. IEEE Sens. J. 2019, 19, 7179-7191. [CrossRef]

5. Liu, Z.; Xiao, H.; Liao, M.; Han, X.; Chen, W.; Zhao, T.; Jia, H.; Yang, J.; Tian, Y. PDMS-Assisted Microfiber M-Z Interferometer With a Knot Resonator for Temperature Sensing. IEEE Photon. Technol. Lett. 2019, 31, 337-340. [CrossRef]

6. Zhang, Q.; Lei, J.; Chen, Y.; Wu, Y.; Chen, C.; Xiao, H. 3D Printing of All-Glass Fiber-Optic Pressure Sensor for High Temperature Applications. IEEE Sens. J. 2019, 19, 11242-11246. [CrossRef]

7. Lu, L.; Jiang, Z.; Hu, Y.; Zhou, H.; Liu, G.; Chen, Y.; Luo, Y.; Chen, Z. A portable optical fiber SPR temperature sensor based on a smart-phone. Opt. Express 2019, 27, 25420-25427. [CrossRef]

8. Zhu, Z.; Liu, L.; Liu, Z.; Zhang, Y.; Zhang, Y. Surface-plasmon-resonance-based optical-fiber temperature sensor with high sensitivity and high figure of merit. Opt. Lett. 2017, 42, 2948-2951. [CrossRef] [PubMed]

9. Xu, O.; Zhang, J.; Deng, H.; Yao, J. Dual-frequency Optoelectronic Oscillator for Thermal-Insensitive Interrogation of a FBG Strain Sensor. IEEE Photon. Technol. Lett. 2017, 29, 357-360. [CrossRef]

10. Murray, M.J.; Davis, A.; Kirkendall, C.; Redding, B. Speckle-based strain sensing in multimode fiber. Opt. Express 2019, 27, 28494-28506. [CrossRef] [PubMed]

11. Fan, H.; Chen, L.; Bao, X. Combined compression-tension strain sensor over $1 \mu \varepsilon-20 \mathrm{~m} \varepsilon$ by using non-uniform multiple-core-offset fiber. Opt. Lett. 2020, 45, 3143-3146. [CrossRef] [PubMed]

12. Liao, C.R.; Hu, T.Y.; Wang, D.N. Optical fiber Fabry-Perot interferometer cavity fabricated by femtosecond laser micromachining and fusion splicing for refractive index sensing. Opt. Express 2012, 20, 22813-22818. [CrossRef] [PubMed]

13. Ouyang, Y.; Xu, X.; Zhao, Y.; Zhou, A.; Yuan, L. Temperature Compensated Refractometer Based on Parallel Fiber Fabry-Pérot Interferometers. IEEE Photon. Technol. Lett. 2018, 30, 1262-1265. [CrossRef]

14. Wei, T.; Han, Y.; Li, Y.; Tsai, H.-L.; Xiao, H. Temperature-insensitive miniaturized fiber inline Fabry-Perot interferometer for highly sensitive refractive index measurement. Opt. Express 2008, 16, 5764-5769. [CrossRef] [PubMed]

15. Ni, W.; Lu, P.; Fu, X.; Zhang, W.; Shum, P.P.; Sun, H.; Yang, C.; Liu, D.; Zhang, J. Ultrathin graphene diaphragm-based extrinsic Fabry-Perot interferometer for ultra-wideband fiber optic acoustic sensing. Opt. Express 2018, 26, 20758-20767. [CrossRef] [PubMed]

16. Fan, H.; Zhang, L.; Gao, S.; Chen, L.; Bao, X. Ultrasound sensing based on an in-fiber dual-cavity Fabry-Perot interferometer. Opt. Lett. 2019, 44, 3606-3609. [CrossRef] [PubMed]

17. Fan, H.; Chen, L.; Bao, X. Fiber-Optic Ultrasound Transmitter Based on Multi-Mode Interference in Curved Adhesive Waveguide. IEEE Photon. Technol. Lett. 2020, 32, 325-328. [CrossRef]

18. Fan, H.; Chen, L.; Bao, X. Chalcogenide microfiber-assisted silica microfiber for ultrasound detection. Opt. Lett. 2020, 45, 1128-1131. [CrossRef]

19. Yu, F.; Xue, P.; Zhao, X.; Zheng, J. Simultaneous Measurement of Refractive Index and Temperature Based on a Peanut-Shape Structure In-Line Fiber Mach-Zehnder Interferometer. IEEE Sens. J. 2019, 19, 950-955. [CrossRef]

20. Ahmed, F.; Ahsani, V.; Melo, L.; Wild, P.; Jun, M.B.G. Miniaturized Tapered Photonic Crystal Fiber Mach-Zehnder Interferometer for Enhanced Refractive Index Sensing. IEEE Sens. J. 2016, 16, 8761-8766. [CrossRef] 
21. Arjmand, M.; Chiavaioli, F.; Berneschi, S.; Baldini, F.; Soltanolkotabi, M.; Trono, C. Effect of induced inner curvature on refractive index sensitivity in internally tilted long-period gratings. Opt. Lett. 2016, 41, 1443-1446. [CrossRef] [PubMed]

22. Shen, F.; Wang, C.; Sun, Z.; Zhou, K.; Zhang, L.; Shu, X. Small-period long-period fiber grating with improved refractive index sensitivity and dual-parameter sensing ability. Opt. Lett. 2017, 42, 199-202. [CrossRef]

23. Gauvreau, B.; Hassani, A.; Fehri, M.; Kabashin, A.; Skorobogatiy, M. Photonic bandgap fiber-based Surface Plasmon Resonance sensors. Opt. Express 2007, 15, 11413-11426. [CrossRef]

24. Rifat, A.A.; Haider, F.; Ahmed, R.; Mahdiraji, G.A.; Adikan, F.R.M.; Miroshnichenko, A.E. Highly sensitive selectively coated photonic crystal fiber-based plasmonic sensor. Opt. Lett. 2018, 43, 891-894. [CrossRef] [PubMed]

25. Liu, C.; Su, W.; Liu, Q.; Lu, X.; Wang, F.; Sun, T.; Chu, P.K. Symmetrical dual D-shape photonic crystal fibers for surface plasmon resonance sensing. Opt. Express 2018, 26, 9039-9049. [CrossRef]

26. Melo, L.B.; Rodrigues, J.M.M.; Farinha, A.S.F.; Marques, C.A.; Bilro, L.; Alberto, N.; Tomé, J.P.C.; Nogueira, R.N. Concentration sensor based on a tilted fiber Bragg grating for anions monitoring. Opt. Fiber Technol. 2014, 20, 422-427. [CrossRef]

27. Leal-Junior, A.; Avellar, L.; Frizera, A.; Marques, C. Smart textiles for multimodal wearable sensing using highly stretchable multiplexed optical fiber system. Sci. Rep. 2020, 10, 13867. [CrossRef]

28. Singh, R.; Kumar, S.; Liu, F.-Z.; Shuang, C.; Zhang, B.; Jha, R.; Kaushik, B.K. Etched multicore fiber sensor using copper oxide and gold nanoparticles decorated graphene oxide structure for cancer cells detection. Biosens. Bioelectron. 2020, $168,112557$. [CrossRef] [PubMed]

29. Luo, H.; Sun, Q.; Li, Y.; Liu, D.; Zhang, L. Highly Birefringent D-Shaped Microfiber and Its Application in High-Sensitive Optical Sensing. IEEE Sens. J. 2016, 16, 4793-4797. [CrossRef]

30. Gomes, A.D.; Frazão, O. Microfiber Knot with Taper Interferometer for Temperature and Refractive Index Discrimination. IEEE Photon. Technol. Lett. 2017, 29, 1517-1520. [CrossRef]

31. Ding, Z.; Sun, K.; Liu, K.; Jiang, J.; Yang, D.; Yu, Z.; Li, J.; Liu, T. Distributed refractive index sensing based on tapered fibers in optical frequency domain reflectometry. Opt. Express 2018, 26, 13042-13054. [CrossRef]

32. Fan, H.; Ma, W.; Chen, L.; Bao, X. Ultracompact twisted silica taper for $20 \mathrm{kHz}$ to $94 \mathrm{MHz}$ ultrasound sensing. Opt. Lett. 2020, 45, 3889-3892. [CrossRef] [PubMed]

33. Fan, H.; Zhang, X.; Zhao, J.; Li, S.; Hua, S.; Zhao, M.; Hu, Y.; Wan, W.; Zhai, Y.; Wen, J.; et al. Controllable coupling between an ultra-high-Q microtoroid cavity and a graphene monolayer for optical filtering and switching applications. Opt. Express 2020, 28, 7906-7916. [CrossRef] [PubMed]

34. Ma, W.; Fan, H.; Chen, L.; Bao, X. Tapered Assisted Dual Micro-Bubble-Device for Ultrasound Sensor. IEEE Photon. Technol. Lett. 2020, 32, 1219-1222. [CrossRef]

35. Yang, S.; Astratov, V.N. Photonic nanojet-induced modes in chains of size-disordered microspheres with an attenuation of only 0.08dB per sphere. Appl. Phys. Lett. 2008, 92, 261111. [CrossRef]

36. Darafsheh, A.; Astratov, V.N. Periodically focused modes in chains of dielectric spheres. Appl. Phys. Lett. 2012, 100, 061123. [CrossRef] [PubMed]

37. Wu, M.-H.; Whitesides, G.M. Fabrication of arrays of two-dimensional micropatterns using microspheres as lenses for projection photolithography. Appl. Phys. Lett. 2001, 78, 2273-2275. [CrossRef]

38. Farahi, K.W.N.; Li, Y.; Limberopoulos, N.I.; Walker, D.E.; Urbas, A.M.; Astratov, V.N. Overcoming the diffraction limit of imaging nanoplasmonic arrays by microspheres and microfibers. Opt. Express 2015, 23, 24484-24496.

39. Ferreira, M.S.; Santos, J.L.; Frazão, O. Silica microspheres array strain sensor. Opt. Lett. 2014, 39, 5937-5940. [CrossRef] [PubMed] 